\title{
Big Data: Horrorszenario oder grosse Chance?
}

Felicitas Witte

Auf dem 11. Schweizerischen Kongress für Gesundheitsökonomie und Gesundheitswissenschaften* diskutierten Experten Vor- und Nachteile der riesigen Datenmengen, die wir alle sammeln. Wir sind schon mitten in der digitalen Revolution, so das Fazit. Doch es gibt Wege, der digitalen Abhängigkeit zu entkommen.

Das Mobiltelefon ist schon wieder zu klein geworden. Vielmehr der Speicher. Kennen Sie das Problem auch? Waren wir früher stolz auf unseren Speicher mit acht Gigabyte, überlegen wir heute, ob der 64 Gigabyte Speicher ausreicht oder ob es nicht besser der 128er sein soll. Ob Fotos, Apps, Mail-Anhänge: Wir generieren immer mehr Daten. Gleichzeitig verlieren wir den Überblick: Brauchen wir wirklich alle 199 Bilder vom Geburtstag der Tante von vor vier Jahren? Oder die 135 im Passbook gespeicherten Flugtickets? Die Dutzende aufgezeichnete Jogging-Touren? Zeit, Unwichtiges in den Papierkorb zu werfen, nimmt sich kaum jemand. In der Medizin sind wir in einer ähnlichen Situation. Mit Hilfe neuer Technologien ist es Forschern in den vergangenen Jahren gelungen, immer mehr Daten zu sammeln: Informationen über unsere Gene ( $\mathrm{Ge}$ nom»), über die in einer Zelle hergestellten RNA-Moleküle («Transkriptom») und Proteine («Proteom»), über Stoffwechselprodukte («Metabolom») oder über die Gesamtheit unserer Mikroorganismen («Mikrobiom»). Daneben natürlich die «altbekannten» Parameter wie Blutdruck, Herzfrequenz oder Laborwerte, Hirnströme oder Ergebnisse von Aufnahmen des Körpers. Doch unser «Medizin-Arbeitsspeicher» kommt an seine Grenzen. Wer soll all die Daten verwalten? Wer entscheidet, was wichtig ist und was nicht? Wie können wir die Informationen nutzen? «Big Data» bezeichnen Experten diese Datenmengen, die zu gross sind, um sie mit klassischen Methoden zu verarbeiten und auszuwerten.

Wir können uns Big Data nicht mehr entziehen, wir sind schon mitten drin - das war das Fazit der Experten auf dem 11. Schweizerischen Kongress für Gesundheitsökonomie und Gesundheitswissenschaften, der kürzlich in Bern stattfand.

«Die digitale Transformation ist auf dem Weg», sagte Thomas Gauthier, Professor für Strategie an der Universität für angewandte Wissenschaften in Genf. «Das kann Angst machen, ist aber auch eine grosse Chance.» Inzwischen könne man fast jeden Parameter selbst bestimmen oder einfach bestimmen lassen. Die Messungen werden zudem immer billiger und unsichtbarer. So kann man heutzutage etwa schon für wenige Hundert Franken sein gesamtes Genom screenen lassen, Armbänder dokumentieren unauffällig die Herzfrequenz und sogar der Blutzucker lässt sich inzwischen nichtinvasiv bestimmen über eine Kontaktlinse. Bis vor kurzem hat der Arzt die Daten erho-

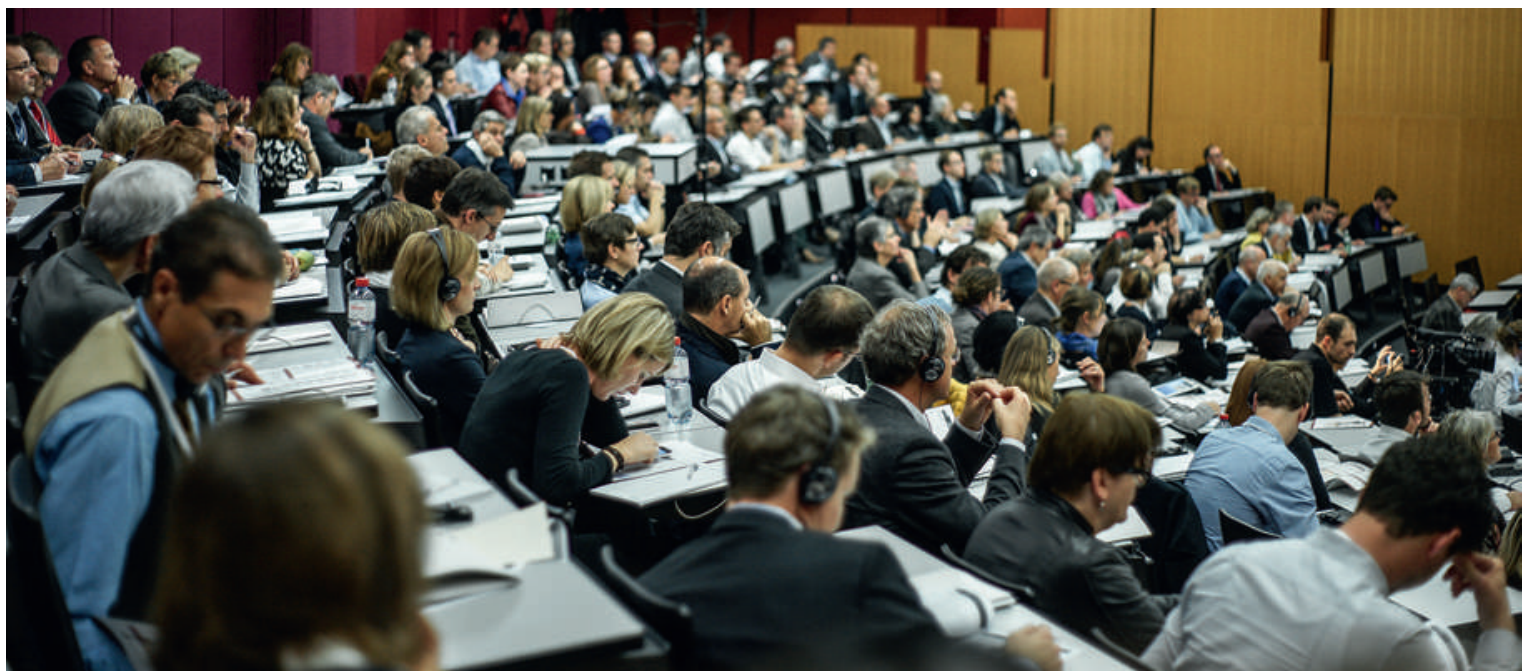

Wichtiges Thema auf dem Berner Kongress: «Big Data»-Datenmengen, die zu gross sind, um sie mit klassischen Methoden zu verarbeiten und auszuwerten. 
ben, gesammelt und interpretiert. «Jetzt sollen Patienten ihre Gesundheit selbst in die Hand nehmen", sagte Gauthier. "Sie sammeln ihre Daten, bewirtschaften sie selbst und treffen Gesundheitsentscheidungen - das interessiert natürlich Google, Amazon \& Co.» Jeder trägt einen kleinen «Bach» an Daten bei, der später zu einem grossen «Datenfluss» wird, Big Data eben.

Schon lange werden klinische Daten in Studien gesammelt. Jetzt werden die Daten «öffentlich», jeder kann sie selbst erheben und speichern. Immer mehr Menschen tauschen sich zudem in Online-Patientenforen aus, die quasi als «neue klinische Studienplattform" fungieren. Gleichzeitig entstehen neue Gesundheitsdienste, die sich eher darauf konzentrieren, das Wohlbefinden zu verlängern und nicht nur eine Krankheit $\mathrm{zu}$ therapieren. Gauthier bezeichnet die neuen Daten als "Echtzeit-Daten", die auf breiter Ebene eingesetzt werden können. «Echtzeit-Daten bieten die Möglichkeit, grosse wissenschaftliche Studien über Krankheit und Gesundheit führen zu können. Wir werden eine gewaltige, transparente Datensammlung haben.» Das berge ein grosses Potential, aber auch Risi-

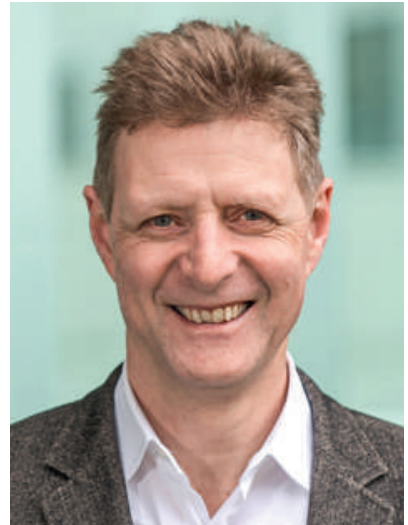

Ernst Hafen vom Institut für Systembiologie der ETH Zürich hat eine genossenschaftlich organisierte Datenbank gegründet. gaben zum Metabolom. Viertens sind die Daten nicht immer verlässlich. «Das liegt zum Beispiel daran, dass Sensoren nicht sensitiv genug sind oder Daten erfasst werden an Orten, wo man sie nicht erfassen kann», erklärte Kaiserswerth. «Den erhobenen Daten kann man nicht ohne weiteres glauben, sie müssen erst einmal validiert werden.» Nicht nur die Daten haben sich verändert, auch die Computer. «Damit sind wir heute in der Lage, riesengrosse Datenmengen zu durchforsten und Erkenntnisse aus diesen Daten zu gewinnen.» Als Beispiel nannte der Informatiker den Watson Discovery Advisor von IBM. Das ist ein "Supercomputer», der in kürzester Zeit gesammelte Daten von Patienten in der Literatur und im Internet durchsucht und Ärzten hilft, ihre Patienten besser zu therapieren. Wird bei einem Patienten mit einem Tumor, etwa Hautkrebs, beispielsweise eine seltene genetische Veränderung gefunden, sucht der Supercomputer Dr. Watson nach einer ähnlichen genetischen Veränderung in seinen Daten. Vielleicht findet er die gleiche Veränderung bei einem $\mathrm{Pa}$ tienten mit einem anderen Krebs, dem ein bestimmtes Medikament geken. Wir müssten uns Gedanken machen, wie wir mit Big Data umgehen wollen, sagte Gauthier, und gab einige strategische Empfehlungen: Zunächst gelte es zu erkennen, wo bereits welche Daten erhoben werden und fliessen, wie sehr bereits das Internet durchdrungen ist. Dann müsse man sich Gedanken machen, wer die Daten verwalte, wer zum Beispiel die Schnittstelle sei zwischen Patienten, Pharmafirmen und BiotechFirmen. Neue Jobs könnten hierbei entstehen, etwa der eines «digitalen Coaches». Diese Aufgabe könnte auch ein Apotheker übernehmen, dessen Beruf jetzt ja schon nicht mehr das sei, was er früher einmal war.

\section{Ohne Systembiologie verlieren wir den Überblick}

Big Data, erklärte Matthias Kaiserswerth, Direktor und Vize-Präsident IBM Research Zürich, beinhalte vier Charakteristika: Erstens wachsen die Daten exponentiell. Zweitens werden sie in Echtzeit generiert und wollen rasch verarbeitet werden. Werde ich über ein pathologisches EKG erst nach Stunden informiert, kann es schon zu spät sein, weil der Infarkt schon stattgefunden hat. Drittens unterscheiden sich die Daten ziemlich voneinander: von klassischen Angaben aus der Patientenakte über Genanalysen oder An- holfen hat. So kann der Arzt versuchen, ob das gleiche Medikament auch bei seinem Patienten mit dem Hautkrebs hilft. Systembiologie nennen Experten dieses neue Forschungsgebiet: Mit Hilfe leistungsstarker Computer versuchen Systembiologen, ein Gesamtbild über das Genom, Transkriptom, Proteom, Metabolom, Mikrobiom und andere Prozesse im Organismus zu bekommen. "Je mehr Daten wir haben, desto schwieriger wird es für den Arzt, den Überblick zu behalten», sagte Kaiserswerth. "Das ist das Grundproblem von Big Data.» Hier könne die Systembiologie jedoch wertvolle Dienste leisten: «Weiss der Arzt zum Beispiel wegen der Menge an Informationen nicht, welches Medikament er bei einem bestimmten Krebs auswählen soll, könnte der Supercomputer ihm das richtige Medikament vorschlagen und ihm auch noch erklären, warum er dazu rät», erklärt Kaiserswerth. Gibt der Arzt später ein, dass das Medikament geholfen hat, «lernt» das der Supercomputer.

\section{Zu viel Datenschutz kann der Forschung im Wege stehen}

Bei diesem Zukunftsszenario aus jederzeit zugängche gefragt haben wie Jacques de Haller, ehemaliger lichen Daten mögen sich manche Zuhörer das Glei- 
Präsident der FMH: «Wie ist denn das Arztgeheimnis mit Big Data vereinbar?» Jetzt habe man endlich die treibende Kraft, das Arztgeheimnis zu lockern beziehungsweise sich damit auseinanderzusetzen, kommentierte Thomas Gauthier in Bern. "Wir sind in der Schweiz zu dogmatisch, was Daten angeht», sagte de Haller. «In den skandinavischen Ländern gibt es schon seit Jahrzehnten Register mit anonymisierten Patientendaten, die eine exzellente Forschung ermöglichen.» Balz Ryf, Leiter Marktzulassung bei Vifor Pharma, erinnerte sich, dass er einmal in Skandinavien gefragt habe, ob niemand Angst habe, seine Daten preiszugeben. "Die haben meine Frage gar nicht verstanden». In der Schweiz gebe es Nachholbedarf, sagte Kaiserswerth. "Wir stehen uns selbst im Wege. Es gibt genügend Techniken, wie man für sicheren Datenschutz sorgen kann. Man muss nur lernen, sie zu verstehen und anzuwenden.» Um dieses Problem weiter zu diskutieren, organisiert Jacques de Haller am 3. Februar 2015 einen Workshop zum Thema "Transparenz von Patientendaten: Wie viel Datenschutz ist ungesund?» (mehr Infos unter www.forumsante.ch).

Eva van Pelt, General Manager Healthcare und Life Sciences bei der Elektrotechnik-Firma Hitachi, machte mit anschaulichen Vergleichen deutlich, wie «big» eigentlich Big Data sind. Im Jahr 2011 produzierten wir weltweit 1,8 Zettabytes Daten. «Wer sich darunter nicht auf Anhieb etwas vorstellen kann: Das entspricht 200 Milliarden HD-Filmen mit einer Dauer von zwei Stunden pro Film», erklärte van Pelt. «Ein Mensch würde 47 Millionen Jahre benötigen, um diese anzusehen.» Diese Datenmenge werde sich voraussichtlich jedes Jahr verdoppeln. "Alle diese Daten nutzen wir von der Wiege bis zum Grab", sagte van Pelt und fügte schmunzelnd hinzu: «Das wollen zumindest Firmen wie Hitachi.» Die Zukunft habe aber schon begonnen, Big Data habe längst Einzug gehalten in das Gesundheitswesen: Zum einen werde die Medizin immer personalisierter. So bestimmen Ärzte heute bei vielen Tumoren Genveränderungen und passen die Therapie individualisiert an, etwa die Gabe des Antikörpers Trastuzumab bei einer Frau mit HER2-positivem Brustkrebs. Zum anderen wird die Medizin präziser, etwa weil man mit computerbasierten Analysen eine genauere Vorhersage machen kann über die Tumorprogression. Der Patient werde immer mehr partizipieren an seiner Therapie, hofft van Pelt, wenn er zum Beispiel mittels Mobiltelefon seine eigenen Krankheitsdaten erfasst, sieht und gemeinsam mit dem Arzt analysiert. Durch die Analyse von Daten und persönlichen Risikofaktoren ist es immer besser möglich, das Risiko für Diabetes oder kardiovaskuläre Krankheiten zu schätzen. Es lassen sich zudem durch Kenntnis der molekularen Vorgänge in den Zellen gezielter wirkende Medikamente entwickeln.

\section{Raus aus der digitalen Abhängigkeit}

Big Data berge aber auch einige Herausforderungen, so van Pelt. Man solle den Datenschutz nicht übertreiben. «Das sollte nicht dafür herhalten, die Vorteile von Big Data nicht zu nutzen.» Problematischer findet sie die ethischen Probleme. "Wollen wir alle Mädchen screenen, ob sie irgendwann einmal Brustkrebs bekommen? Als Mitarbeiterin der Industrie habe ich dazu keine Meinung. Als Privatperson aber schon. Ich möchte selbst entscheiden, ob ich das machen möchte.» Diesen Diskussionen müsse die Gesellschaft sich stellen.

Gibt der Arzt später ein, dass das Medikament geholfen hat, "lernt» das der Supercomputer.

Wir müssten gemeinsam bestimmen, wo die Big Data-Reise hingehen soll, und die aktive Beteiligung der Bürger sei zwingend, so Ernst Hafen vom Institut für Systembiologie an der ETH Zürich. «Der Bürger sollte sagen: 〈Ja, ich will einen Beitrag leisten, aber ich will auch etwas dafür haben.»" Google wisse inzwischen mehr über uns als der Arzt. «Das ist die digitale Abhängigkeit oder Leibeigenschaft, in die wir uns begeben haben.» Wir haben keine Kontrolle mehr, was damit passiert, und wir haben nichts von den Werten, die damit erwirtschaftet werden. «Der Zug ist abgefahren. Wir können es jetzt entweder Google, Amazon \& Co. überlassen, oder wir können zu einer digitalen Selbstbestimmung zurückfinden.» Hafen spricht von Datengenossenschaften. Dort sollen wir alle unsere persönlichen Daten selbst verwalten und können dann die Profite teilen. Deshalb gründete er mit anderen Experten 2012 den Verein "Daten und Gesundheit», dessen Ziel genossenschaftlich organisierte Banken für persönliche Daten sind. Die Bürger entscheiden, welche Daten sie mit wem, zu welchem Zweck und wann teilen, und die Mitglieder legen fest, wie die Gewinne aus der Zweitnutzung von Daten - also durch Verkauf an Dritte investiert werden. «Das Projekt Digitale Selbstbestimmung als erstes Land zu realisieren, würde der Schweiz gut stehen», sagte Hafen. "Abgesehen davon ist es zwingend notwendig, dass wir es umsetzen. Sonst geraten wir immer weiter in die digitale Abhängigkeit.» 